\title{
Prevalence of drug interactions in hospitalised elderly patients: a systematic review
}

\author{
Luciana Mello de Oliveira 다 , ${ }^{1}$ Juliana do Amaral Carneiro Diel, ${ }^{1}$ Alessandra Nunes, ${ }^{2}$ \\ Tatiane da Silva Dal Pizzol ${ }^{1,2}$
}

- Additional material is published online only. To view please visit the journal online (http://dx.doi.org/ 10.1136/ejhpharm-2019002111)

'Programa de Pós-Graduação em Epidemiologia, Federal University of Rio Grande do Sul, Porto Alegre, Brazil ${ }^{2}$ Faculdade de Farmácia, Universidade Federal do Rio Grande do Sul, Porto Alegre, Rio Grande do Sul, Brazil

\section{Correspondence to}

Dr Luciana Mello de Oliveira, Programa de Pós-Graduação em Epidemiologia, Federal University of Rio Grande do Sul, Porto Alegre 90040-060, Brazil; oliveiralm@gmail.com

Received 9 October 2019 Revised 13 January 2020 Accepted 16 January 2020 Published Online First 10 February 2020

\section{EAHP Statement 5: Patien} Safety and Quality Assurance.

Check for updates

(C) European Association of Hospital Pharmacists 2021. No commercial re-use. See rights and permissions. Published by BMJ.

To cite: de Oliveira LM, Diel JdAC, Nunes A, et al Eur J Hosp Pharm

2021:28:4-9.

\section{ABSTRACT}

Background The prevalence of drug-drug interactions (DDIs) in hospital settings is variable, and elderly patients are considered a high risk population for DDIs. There are no systematic reviews describing the prevalence of DDIs in hospitalised elderly patients.

Objectives To assess and summarise the available data on the prevalence of DDIs in hospitalised elderly patients and to describe which drugs, drug classes and drug combinations are most commonly involved in DDIs.

Data source A systematic electronic literature search was conducted on Medline/PubMed, Embase, Lilacs, SciElo, Web of Science, Cinahl, Scopus, Cochrane, OpenGrey, Capes Thesis Bank, OasisBR, OpenAire and abstracts from scientific events, without limitation on language or period of publication. Study selection was completed on 21 September 2018.

Study eligibility criteria, participants and interventions Original observational studies that reported the prevalence of actual or potential DDIs during hospitalisation in patients aged 60 years or older were included. The main outcome measure was prevalence of DDIs and number of DDIs per patient. Subgroup analysis was performed in studies that reported the prevalence of DDIs in geriatric units. Study appraisal and synthesis methods Study quality was assessed using the Agency for Healthcare Research and Quality methodological checklist for cross sectional and prevalence studies.

Results 34 studies were included, involving 9577 patients. The prevalence of DDIs ranged from $8.34 \%$ to $100 \%$. In studies conducted in geriatric units, the prevalence ranged from $80.5 \%$ to $90.5 \%$. The number of DDls per patient ranged from 1.2 to 30.6 . Single drugs most commonly involved in DDIs were furosemide, captopril, warfarin and dipyrone. Drug classes mostly involved were potassium sparing diuretics and angiotensin converting enzyme inhibitors.

Limitations The main limitation is the heterogeneity between the included studies that precluded a metaanalysis. Several different methods were used to identify DDIs, majorly, and potential DDIs. Few studies have reported measures to control the quality of the collected data.

Conclusions and implications of key findings The prevalence of DDIs ranged widely, and the variation may reflect differences in the conditions of the elderly patients and level of attention (or complexity of care), as well as methodological differences, especially the methods and/ or software used to identify DDIs.

Systematic review registration number CRD42018096720

\section{INTRODUCTION}

A drug-drug interaction (DDI) is described as the ability of one drug to enhance, diminish and/or modify the action or effects of another drug when administered successively or simultaneously. ${ }^{1}$ DDIs are a particularly important type of adverse drug event as they can alter drug effectiveness and security. ${ }^{2}$ Although not always avoidable, DDIs are often predictable. ${ }^{3}$ Pharmacodynamic DDIs are those related to the pharmacological activity of interacting drugs, through direct effect on receptor function, interference with a biological or physiological control process or additive/opposed pharmacological effect, and are less frequent. Pharmacokinetic DDIs are related to the effects of one drug on the absorption, distribution, metabolism or excretion of another drug, and can modify drug concentrations levels, leading to ineffectiveness or toxicity, and are more prevalent than pharmacodynamics DDIs. ${ }^{4}$ Considering mechanisms and prevalence, pharmacokinetic DDIs have a higher impact on patient safety than pharmacodynamic DDIs.

Actual DDIs are identified from patient adverse outcomes whereas potential DDIs are those identified through analysis of the pharmacokinetic and pharmacodynamics profiles of each drug in use, and identification of possible adverse events due to the association. ${ }^{5}$ Since a potential DDI may or may not result in an adverse outcome, the incidence of actual DDIs in the literature is consistently lower compared with potential DDIs, ${ }^{6}$ and therefore each patient should be evaluated individually, considering the risk-benefit ratio. ${ }^{2}$

The prevalence of DDIs in the hospital setting is variable. Studies conducted with hospitalised patients in different clinical settings have shown a prevalence of $5.3-83.9 \% .^{7-12}$ It is estimated that up to two-thirds of intensive care patients experience at least one potential DDI during their hospital stay. ${ }^{13}$ It seems that the majority of prescribed interacting drug combinations involve a limited number of drugs, ${ }^{14}$ and the main mechanism of DDIs is pharmacokinetic, involving drug metabolism via cytochrome P450. ${ }^{15}$

Elderly patients are considered a high risk population for DDIs. ${ }^{16}$ The prevalence of DDIs in elderly outpatients with multimorbidity was recently reported to be between $25.1 \%$ and $100 \%$, where the number of DDIs per 100 patient ranged from 30 to $388.3 .{ }^{17}$ The incidence of DDIs during hospital stay is not well defined. Ageing is an independent risk factor for DDIs, ${ }^{18}$ as elderly patients tend to receive more medications than other ages group as a consequence of the many physiological changes related 
to the ageing process and the accompanying health problems. ${ }^{19}$ Therefore, in this population, potential DDIs are often unavoidable. However, the risk of adverse outcomes due to DDIs seem to be particularly serious in the elderly. A systematic review has shown that DDIs were responsible for $0.57 \%$ of hospital admissions in general but for $4.8 \%$ of elderly patients. ${ }^{20}$

To date, no systematic review has specifically addressed the prevalence of DDIs in elderly patients in an inpatient setting. The aim of this systematic review was to collate the evidence about actual and potential DDI prevalence in the hospitalised elderly patient obtained from observational studies. We also wanted to describe which drugs are most commonly involved in DDIs in this population, as well the methods used to identify DDIs in elderly patients.

\section{METHODS}

\section{Protocol, registration and ethics}

The protocol for this review was registered with PROSPERO (https://www.crd.york.ac.uk/prospero/, registration No CRD42018096720). ${ }^{21}$ No ethics committee approval was sought, as this study is a systematic review of literature. This work was performed in accordance with the Preferred Report Items for Systematic Reviews and Meta-Analysis (PRISMA) statement for reporting systematic reviews and meta-analyses of observational studies. ${ }^{22}$

\section{Eligibility criteria}

Observational studies (cohort, cross sectional and case control studies), reported as original articles, thesis and abstracts from scientific events and meetings, published at any time, in any language, that reported actual or potential harmful DDIs during hospitalisation in elderly patients, were included. When the information provided in the original document was not sufficient to collect or calculate DDI rates, additional specific data were requested through electronic contact with the authors and feedback.

Elderly was defined as aged 60 years or older. Most developed countries have accepted the chronological age of 65 years as a definition of elderly or older person, but in developing countries 60 years of age is adopted. ${ }^{23}$

Studies were excluded if they: (a) focused on DDIs involving a single drug, drug class, a few specific drugs or only drugs that could cause a specific clinical presentation; (b) presented data from a narrow disease population; (c) evaluated rates of DDIs on prescriptions, rather than in patients; (d) reported DDIs at hospital admission and discharge only; (e) reported interventions on DDIs but not the rates before intervention; (f) analysed the prevalence of DDIs in adults, including the elderly, but did not provide enough data to calculate the prevalence of DDIs in the elderly population of the study, in the original document or after the information was requested; (g) reported data from elderly inpatients and outpatients, but there was insufficient data to extract the rates in the hospital setting from the study and/or after requested; and (h) had not intended to assess prevalence, but only to characterise DDIs in the population of interest.

\section{Information sources and search strategy}

$\mathrm{MeSH}$ terms and key words were used to search databases. Medline/PubMed, Embase, Lilacs, SciElo, Web of Science, Cinahl, Scopus, Cochrane, OpenGrey, Capes Thesis Bank, OasisBR, OpenAire and abstracts from scientific events were searched from inception to 3 June 2018 to identify potential articles and theses. We screened the lists of references of the

\section{Box 1 Full search strategy used in MEDLINE}

\#1. epidemiology OR epidemiology [MeSH Terms] OR epidemiology [mh] OR epidemiology [tw] \#2. pharmacoepidemiology OR pharmacoepidemiology[MeSH Terms] OR pharmacoepidemiology[mh] OR pharmacoepidemiology[tw] OR prevalence OR prevalence[MeSH Terms] OR prevalence[mh] OR prevalence[tw] \#3. drug interactions OR drug interactions[MeSH Terms] OR drug interactions[mh] OR drug interactions[tw] \#4. drug related side effects and adverse reactions OR drug related side effects and adverse reactions [MeSH Terms] OR drug related side effects and adverse reactions[mh] OR drug related side effects and adverse reactions[tw] \#5. aged OR aged [MeSH Terms] OR aged[mh] OR aged[tw] \#6. aged, 80 and over OR aged, 80 and over [MeSH Terms] OR aged, 80 and over[mh] OR aged, 80 and over[tw]

\#7. \#1 OR \#2

\#8. \#3 OR \#4

\#9. \#5 OR \#6

\#10. \#7 AND \#8

\#11. \#9 AND \#10

included studies to check for other possible studies. When more than one publication presented the same patient data, the most completed one was included. The full search strategy used in Medline/PubMed is described in Box 1.

\section{Study selection}

A standard form was used to select publications. First, two authors (LMO and JACD) independently screened titles and abstracts identified in all sources and selected articles that seemed to meet the inclusion criteria. Then, previously included documents had their full text independently evaluated and selected by two authors (LMO and JACD) to reduce the subjectivity of the selection process. Any discrepancies were resolved by discussion with a third researcher (TSP). Study selection was finished on 21 September 2018 .

\section{Data collection process}

Two reviews (LMO and JACD) independently extracted data using a standard form. Disagreements between reviewers in data collection were resolved by consensus and the opinion of a third reviewer (TSP) if necessary. Relevant missing information, such as prevalence of DDIs in elderly patients (or raw data for calculation) of studies conducted in adult patients, was requested from the original authors. Also, information on number of DDIs per patient, data on severity of DDIs, drugs, drug classes and drug combinations mostly involved in DDIs were requested. The data collection process was finished on 23 January 2019.

\section{Outcome measures (summary measures)}

The main outcome measure was (i) prevalence of actual or potential DDIs, calculated as the percentage of patients that presented at least one DDI during their hospital stay among the total number of patients studied. When possible, DDI prevalence rates were extracted; however, in a number of cases, prevalence rates were calculated from the information reported. Other information extracted, if available, included: (ii) number of DDIs per patient, defined as the number of DDIs 
divided by the number of patients with at least one DDI. When available, data were extracted on (iii) the most common drugs involved with DDIs, (iv) the most common combinations of drugs involved in DDIs and (v) the methods to identify DDIs.

\section{Synthesis of results}

As study designs, participants, interventions and/or mainly the reported outcome measures (DDI prevalence) were not provided by the authors, we focused on describing the studies, their results and on qualitative synthesis rather than on a meta-analysis.

\section{Risk of bias in individual studies (quality assessment)}

Due to the cross sectional nature of the studies included, study quality was assessed using the Agency for Healthcare Research and Quality (AHRQ) methodological checklist for cross sectional and prevalence studies ${ }^{24-26}$ This assessment tool is an 11 item questionnaire to explore the quality of data collection, inclusion criteria, outcome measurement and other measurements (see online supplementary table 1 ). Items were answered as yes (+), no (-), unclear (U) and not applicable (NA) to the study.

Quality assessment was conducted by LMO and JACD. Disagreements between reviewers were resolved by consensus and the opinion of a third reviewer (TSP) if necessary. Study quality was not an exclusion criteria. The quality assessment process was finished on 1 March 2019.

\section{Risk of bias across studies}

Risk of bias across studies, particularly publication bias and selective reporting bias, was not evaluated because these types of biases are unlikely for the predominantly descriptive research question.

\section{Additional analyses}

A post hoc analysis was conducted in geriatric units to assess differences in prevalence in this subgroup to all aged population.

\section{RESULTS}

\section{Study selection}

After the database search, 2409 documents were identified. After duplicates removal (337 documents removed) and screening by title and abstract (1839 documents removed), 233 remained for full reading and evaluation. After full text reading, 76 documents were considered for inclusion. Of those, 46 studies reported data on DDIs in adults, and the authors were approached with a request for specific data on the prevalence of DDIs in the elderly patients only. In addition to the prevalence of DDIs in the elderly (or raw data for calculation), information about number of DDIs per patient, data on severity of DDIs, drugs, drug classes and drug combinations mostly involved in DDIs were requested. Only three authors replied to our emails. Hence 34 were included in this review, as shown in figure 1 .

\section{Study characteristics}

The studies included were published between 2003 and 2018 . Sample size varied from 44 to 1510 , totalling 9577 patients. The majority of papers were cross sectional and from developing countries: 9 studies were from Brazil, ${ }^{27-35} 6$ studies were from India, ${ }^{36-41} 2$ from Mexico, ${ }^{42}{ }^{43} 2$ from Croatia, ${ }^{44} 451$ from Venezuela, ${ }^{46} 1$ from Serbia, ${ }^{47} 1$ from Nepal, ${ }^{48} 1$ from Iran $^{49}$ and one from Ethiopia. ${ }^{50}$ The remaining 10 studies were from Spain, ${ }^{51}{ }^{52}$ Norway, ${ }^{53}$ Portugal, ${ }^{54}$ Germany, ${ }^{55}$ Italy, ${ }^{56}$ Austria, ${ }^{57}$ Israel, ${ }^{58} \mathrm{Canada}^{15}$ and Australia. ${ }^{59}$ Of the studies included, 5 were conducted in geriatric units 32525558 and 15 were conducted in internal medicine units. ${ }^{28} 3033353841$ 44-46 485051535457 Online supplementary table 2 shows the general characteristics, prevalence of DDIs and methods to identify DDIs in each study.

\section{Results of individual studies}

We identified 21 studies that examined DDI rates or provided enough data for its calculation. The prevalence of DDIs ranged from $8.34 \%{ }^{36}$ to $100 \% .{ }^{3746}$ In studies conducted in geriatric units, the prevalence ranged from $80.5 \%$ to $90.5 \%$. In studies

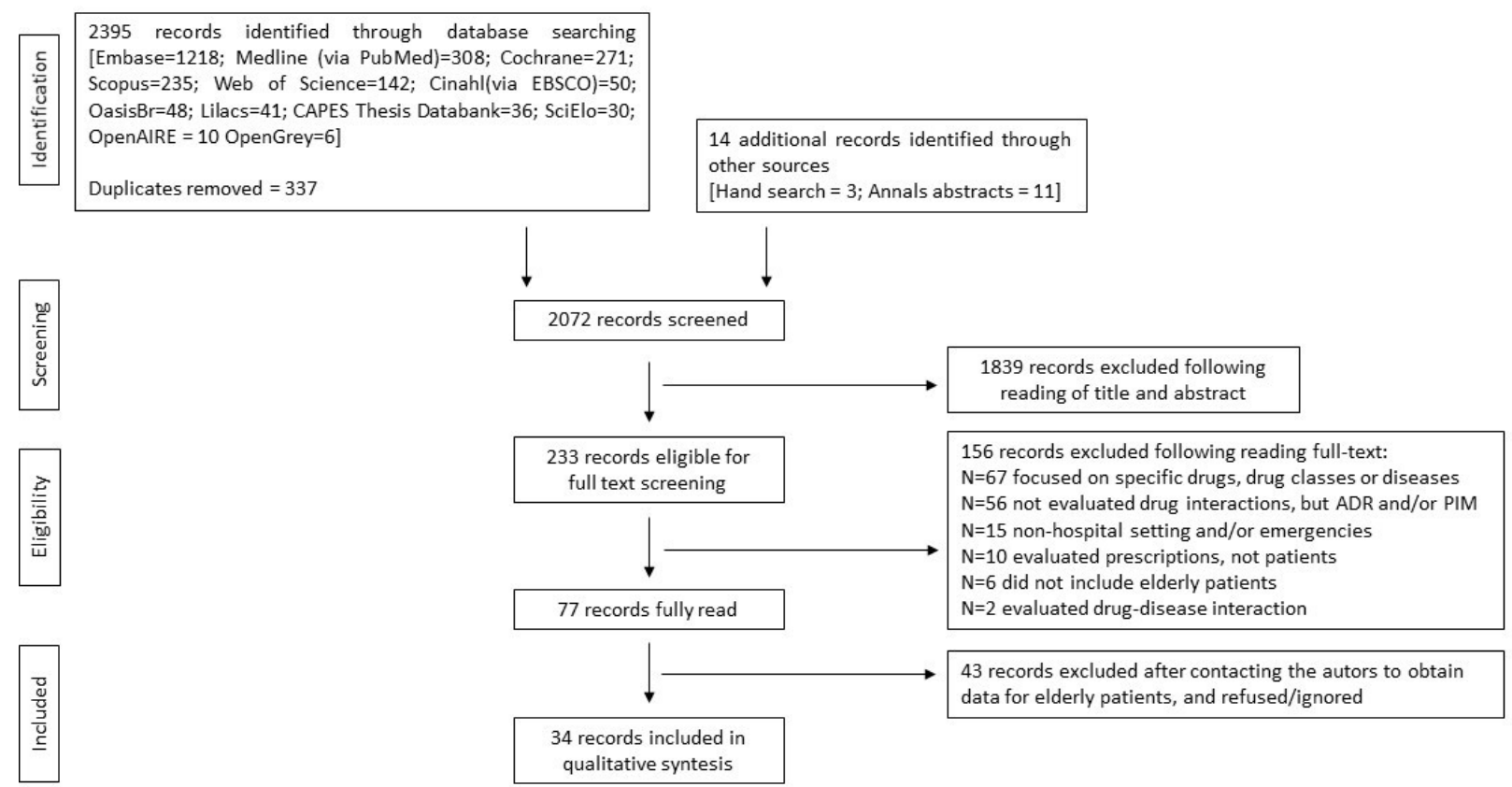

Figure 1 Preferred Report Items for Systematic Reviews and Meta-Analysis (PRISMA) flow chart for study identification and inclusion in a systematic review of the prevalence of drug interactions in hospitalised elderly patients. PIM, potentially inappropriate medication; ADR, adverse drug reaction 
performed in other hospitalisation units, the prevalence ranged from $8.3 \%$ to $100 \%$. The mean number of drugs per patient ranged from 2 to 23.9. Only one study evaluated actual DDIs. ${ }^{53}$ Fifteen studies analysed DDIs through the Drug-Reax System (also known as Micromedex), 27-30 32-36383943475058 five studies analysed DDIs using Beers criteria, 3740414445 four studies analysed DDIs using the Medscape Drug Interaction Checker ${ }^{38}$ 46-48 and two studies used the Epocrates Interaction Check. ${ }^{47} 54$ Ten studies evaluated DDIs with more than one type of soft-

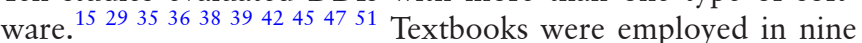
studies. ${ }^{152935383942495159}$ In one case, the source for DDI evaluation was locally adopted lists ${ }^{53}$ and in another, databases that were not explicit. ${ }^{55}$ Online supplementary table 2 presents individual results of each study.

The number of DDIs per patient was reported in only 12 studies, and ranged from 1.2 to 30.6. Although the information provided in the studies did not allow meaningful quantification as the results were ranked using different methodologies and therefore cannot be correlated, the most frequent DDIs were reported in some articles. Single drugs most commonly involved in DDIs were furosemide, presented in three studies, and captopril, warfarin and dipyrone, presented in two studies. Drug classes mostly involved with DDI were potassium sparing diuretics and angiotensin converting enzyme inhibitors, each one reported in two studies. The only study that evaluated actual DDIs, reported that the single drugs most commonly involved in DDIs were warfarin, digitoxin and prednisolone, and the drug classes mostly involved were antithrombotic agents, nonsteroidal anti-inflammatory agents and angiotensin converting enzyme inhibitors. ${ }^{53}$ Regarding drug combinations, drugs that were more frequently described in DDIs were digoxin (10 times), aspirin (9 times), clopidogrel (8 times), metoclopramide, chlorpromazine, ketoconazole, haloperidol, amiodarone and furosemide (7 times each), spironolactone and omeprazole (6 times each), and promethazine, captopril, dipyrone and heparin (5 times each). Anticoagulants was the drug class most frequently described (6 times). The most prevalent drug combinations were digoxin and furosemide, captopril and spironolactone, haloperidol and promethazine (cited three times each), digoxin and metoclopramide, clopidogrel and omeprazole, and aspirin and clopidogrel (cited two times each). Online supplementary table 3 shows the number of patients with interactions, number of DDIs per patient with at least one DDI, drugs or drug classes mostly involved in DDIs and drug combinations mostly involved in DDIs.

\section{Quality assessment and risk of bias in individual studies}

All of the studies included in this review presented cross sectional analysis and therefore questions 10 and 11 of the AHRQ methodological checklist ${ }^{26}$ were not suitable for evaluation. For question 7, a designation of 'not applicable' was recorded when no patient was excluded from the initial sample. One study was reported in Persian, ${ }^{49}$ and another study was an abstract from a scientific meeting. ${ }^{58}$ In both cases, full evaluation was not possible. Complete quality assessment of the reviewed studies and risk of bias can be found in the online supplementary figure 1 .

\section{DISCUSSION}

\section{Summary of evidence}

The prevalence of DDIs ranged widely, from $8.34 \%$ to $100 \%$. The great variation observed is similar to that seen in a systematic review with outpatients $(25.1 \%$ to $100 \%) .{ }^{17}$ Among the possible causes for this wide variation is the number of medications per patient (ranging from 2 to 23.9), differences in the patients' health conditions, level of attention (or complexity of care) in different settings and the methods and software used to detect DDIs. In addition, contrary to our expectations, the prevalence of DDIs in hospitalised elderly was not higher than that observed in the community. Among the elderly hospitalised in geriatric units, the variation was a little lower $(80.5 \%$ to $90.5 \%)$, but with consistently higher rates. These findings should be interpreted with caution since in 13 studies (38\% of all studies included in the systematic review), data on the prevalence of DDIs were not available, making it impossible to summarise the data in a way that would allow a more precise characterisation of the actual prevalence of DDIs in hospitalised elderly. The use of different methods and/or software devices to identify potential DDIs was another factor that may have influenced the variation observed between the studies, as discussed below.

The drugs most involved with DDIs were diuretics, antihypertensive drugs, anticoagulants, cardiac glycoside drugs and antithrombotic agents. As the majority of the studies were conducted in developing countries, dipyrone was also frequently cited. These drugs were expected as they are commonly administered to elderly to treat conditions associated with ageing, and have been described previously to be associated with drug interactions. ${ }^{14}$ Some drugs are of special interest, as the consequences due to the DDIs are of clinical interest, such as extrapyramidal symptoms and neuroleptic malignant syndrome (promethazine, metoclopramide, chlorpromazine, haloperidol), arrhythmias (amiodarone, cardiac glycoside drugs, promethazine, chlorpromazine, haloperidol), inhibition of metabolism of another drug (ketoconazole) and haemorrhages (anticoagulants, antithrombotics and antiplatelet drugs). It is important to point out that combinations of some classes are primarily a problem of inappropriate prescribing rather than DDIs.

Regarding drug combinations, clopidogrel and omeprazole were associated with significantly higher major adverse cardiac events $^{60}$ and should be avoided when possible. But the DDI aspirin and clopidogrel, cited two times as a harmful DDI, is a dual antiplatelet therapy commonly administered to prevent cardiovascular events. ${ }^{61}$ This is an example of how decision support systems can over report DDIs of low clinical relevance that could lead to malpractice, and how professional expertise is crucial in interpreting these results.

The Drug-Reax System, which some authors argue is the most reliable as it has the highest sensitivity, was used to analyse DDIs in the majority of studies. ${ }^{62}$ The high sensitivity in identifying DDIs can overestimate DDIs. Other databases, such as Medscape Drug Interaction Checker, Epocrates Interaction Check, in house software and textbooks, were used. These sources allow the analysis of the combinations of each drug in the pair with the remaining drugs. However, Beers criteria, employed in five studies, includes predefined drug combinations. ${ }^{63}$ Using Beers criteria alone could underestimate DDI rates.

Several DDI screening software programmes that use clinicians' decision support systems are available, but one limitation of is the lack of clinical relevance, which results in over reporting of potential DDIs. ${ }^{64}$ Also, information obtained in one database could be different from another. A systematic review evaluated the usability and appropriateness of several databases and found large discrepancy in the number and relevance of detected DDIs, as high as $81.1 \%$, and only a few studies employed more than one database to evaluate DDIs. ${ }^{65}$ Therefore, the software itself could have modified DDI rates. Ideally, more than one source should be used and the information obtained should be interpreted with clinical knowledge. In this review, only 10 studies 
evaluated DDIs using more than one software product, but only two reported differences between the methods. Doan and colleagues ${ }^{15}$ found rates between $80 \%$ and $97 \%$, and Jankovic ${ }^{47}$ and colleagues found rates between 93.84 and 95.49, depending on the software used. Nevertheless, prevalence rates in these 10 studies ranged from $8.34 \%$ to $98.6 \%$.

\section{Strengths}

Physiological changes and its pharmacokinetic and pharmacodynamic alterations, the development of chronic diseases and accompanied polypharmacy, and the increasing number of therapeutic options put elderly patients at high risk of DDI related adverse events, especially in a hospital environment. This is the first comprehensive systematic review to describe the prevalence of DDIs in hospitalised elderly patients and the drugs commonly involved in these DDIs. We have focused on studies conducted in patient populations and did not include papers which described research on predefined drug pairs.

\section{Limitations}

The main limitation was the heterogeneity between the included studies that precluded a meta-analysis. Several different methods were employed to identify DDIs. Also, papers included in this review described, primarily, potential drug interactions; evaluation of potential DDIs could overestimate DDI rates, as it characterises as a DDI any drug combination that could lead to a DDI every time the two drugs are administered together. Real or actual DDI rates are obtained from clinical evidence. Not every potential DDI translates to a real DDI, which means patient harm. In our systematic review, only one study evaluated real drug interactions, and found a DDI rate of $8.8 \%$. To evaluate potential DDIs could overestimate DDI rates, even though, in this study, potential DDI rates ranged between $8.34 \%$ and $100 \%$. It is estimated that only about $6 \%$ of initially detected DDIs are clinically relevant, and the incidence of potentially serious DDIs is less than $1 \%$ in an ambulatory setting. ${ }^{66}$ Studies that evaluated only real DDIs could lead to information about drug combinations that would produce patient harm. Also, some of the drugs implicated in DDIs are often prescribed, which could lead to a high possibility of causing an interaction.

Currently, there are no gold standard tools for cross sectional studies. The evaluation of methodological quality, based on the AHRQ instrument, showed that the majority of the studies determined the criteria for inclusion and exclusion of patients, sample selection period and data sources used for the research adequately. On the other hand, a few studies reported measures to control the quality of the collected data, such as Blix and colleagues $^{53}$ and Egger and colleagues, ${ }^{55}$ where the identified DDIs were reviewed by a multidisciplinary healthcare team, and Rosas-Carrasco and colleagues, ${ }^{43}$ where the identified DDIs were assessed by geriatricians.

Despite the wide review, which included several databases, grey literature and absence of restrictions such as year of publication and language, reducing the risk of selection/publication bias, 43 studies were excluded from the review due to an inability to obtain specific data. To reduce this bias, as recommended in a previous study, ${ }^{67}$ contacts were made with the authors but the response rate was very low (2 out of 45$)$.

\section{CONCLUSIONS AND IMPLICATIONS FOR CLINICAL PRACTICE}

The prevalence of DDIs was high, and its variation may reflect differences in the elderly conditions and level of attention or complexity of care, as well as methodological differences, especially the methods/software used to identify DDIs. Clinicians must have in mind that different sources will provide different results. Diuretics, antihypertensive drugs, anticoagulants, cardiac glycoside drugs and antithrombotic agents are the drugs commonly administered to elderly to treat conditions associated with ageing and were found to be implicated in the majority of drug interactions. The use of databases with high sensitivity can overestimate DDIs, and the analysis from selected drugs (in the case of using Beers criteria) could underestimate DDI rates. It is important to keep in mind that elderly patients are considered a natural high risk population for DDIs and each patient should be evaluated individually, and in multiple cases DDIs are unavoidable. Ideally, DDIs should be characterised after patient adverse outcomes, and the evaluation of potential DDIs should be used to estimate the risk-benefit ratio. More than one source of information should be used and clinical knowledge should be taken into account when interpreting the results. Future perspectives from this research could compare DDI rates in developed and low-middle income countries, and in different databases, aiming to characterise the best method of identifying DDIs.

\section{Twitter Luciana Mello de Oliveira @oliveiralm}

Contributors LMO and TSP were responsible for the conception of the work. LMO, JACD, AN and TSP were responsible for data collection. LMO and TSP were responsible for data analysis and interpretation. LMO was responsible for drafting of the manuscript. JACD, AN and TSP were responsible for critical review of the manuscript.

Funding LMO was funded by Programa Nacional de Pós-Doutorado da Coordenação de Aperfeiçoamento de Pessoal de Nível Superior (PNPD-CAPES) fund. JACD was funded by CAPES fund. AN was funded by Conselho Nacional de Desenvolvimento Científico e Tecnológico (CNPq) fund.

Competing interests None declared.

Patient consent for publication Not required.

Provenance and peer review Not commissioned; externally peer reviewed.

Data availability statement All data relevant to the study are included in the article or uploaded as supplementary information.

ORCID iD

Luciana Mello de Oliveira http://orcid.org/0000-0002-2065-2001

\section{REFERENCES}

1 Askari M, Eslami S, Louws $M$, et al. Frequency and nature of drug-drug interactions in the intensive care unit. Pharmacoepidemiol Drug Saf 2013;22:430-7.

2 Montané E, Arellano AL, Sanz Y, et al. Drug-related deaths in hospital inpatients: a retrospective cohort study. Br J Clin Pharmacol 2018;84:542-52.

3 Juurlink DNet al. Drug-drug interactions among elderly patients hospitalized for drug toxicity. JAMA 2003;289:1652-8.

4 Palleria C, Di Paolo A, Giofrè C, et al. Pharmacokinetic drug-drug interaction and their implication in clinical management.. J Res Med Sci 2013;18:601-10.

5 Marusic S, Bacic-Vrca V, Obreli Neto PR, et al. Actual drug-drug interactions in elderly patients discharged from internal medicine clinic: a prospective observational study. Eur J Clin Pharmacol 2013;69:1717-24.

6 Magro L, Moretti U, Leone R. Epidemiology and characteristics of adverse drug reactions caused by drug-drug interactions. Expert Opin Drug Saf 2012;11:83-94.

7 Reis AMM, Cassiani SHDB. Adverse drug events in an intensive care unit of a university hospital. Eur J Clin Pharmacol 2011;67:625-32.

8 Sánchez Muñoz-Torrero JF, Barquilla P, Velasco R, et al. Adverse drug reactions in internal medicine units and associated risk factors. Eur J Clin Pharmacol 2010;66:1257-64

9 Davies EC, Green CF, Taylor S, et al. Adverse drug reactions in hospital in-patients: a prospective analysis of 3695 patient-episodes. PLoS One 2009;4:e4439.

10 Kovačević M, Vezmar Kovačević S, Miljković B, et al. The prevalence and preventability of potentially relevant drug-drug interactions in patients admitted for cardiovascular diseases: a cross-sectional study. Int J Clin Pract 2017;71:e13005.

11 Uijtendaal EV, van Harssel LLM, Hugenholtz GWK, et al. Analysis of potential drug-drug interactions in medical intensive care unit patients. Pharmacotherapy 2014:34:213-9.

12 Espinosa-Bosch M, Santos-Ramos B, Gil-Navarro MV, et al. Prevalence of drug interactions in hospital healthcare. Int J Clin Pharm 2012;34:807-17. 
13 Zheng WY, Richardson LC, Li L, et al. Drug-drug interactions and their harmful effects in hospitalised patients: a systematic review and meta-analysis. Eur J Clin Pharmacol 2018;74:15-27.

14 Holm J, Eiermann B, Eliasson E, et al. A limited number of prescribed drugs account for the great majority of drug-drug interactions. Eur J Clin Pharmacol 2014;70:1375-83.

15 Doan J, Zakrzewski-Jakubiak H, Roy J, et al. Prevalence and risk of potential cytochrome P450-mediated drug-drug interactions in older hospitalized patients with polypharmacy. Ann Pharmacother 2013;47:324-32.

16 Mallet L, Spinewine A, Huang A. The challenge of managing drug interactions in elderly people. Lancet 2007;370:185-91.

17 Sánchez-Fidalgo S, Guzmán-Ramos MI, Galván-Banqueri M, et al. Prevalence of drug interactions in elderly patients with multimorbidity in primary care. Int J Clin Pharm 2017;39:343-53.

18 Moura CS, Acurcio FA, Belo NO. Drug-drug interactions associated with length of stay and cost of hospitalization. J Pharm Sci 2009;12:266-72.

19 Hines LE, Murphy JE. Potentially harmful drug-drug interactions in the elderly: a review. Am J Geriatr Pharmacother 2011;9:364-77.

20 Becker ML, Kallewaard M, Caspers PWJ, et al. Hospitalisations and emergency department visits due to drug-drug interactions: a literature review. Pharmacoepidemiol Drug Saf 2007;16:641-51.

21 Oliveira LM, Carneiro JAC, Dal Pizzol TS. Prevalence of drug interactions in the hospitalized elderly: a systematic review. Available: http://www.crd.york.ac.uk/ PROSPERO/display_record.php?ID=CRD42018096720 [Accessed 16 Jan 2019].

22 Liberati A, Altman DG, Tetzlaff J, et al. The PRISMA statement for reporting systematic reviews and meta-analyses of studies that evaluate health care interventions: explanation and elaboration. PLoS Med 2009;6:e1000100.

23 WHO. World report on ageing and health, 2015. Available: http://www.who.int/ ageing/events/world-report-2015-launch/en/ [Accessed 14 Nov 2018].

24 Sanderson S, Tatt ID, Higgins JP. Tools for assessing quality and susceptibility to bias in observational studies in epidemiology: a systematic review and annotated bibliography. Int J Epidemiol 2007;36:666-76.

25 Zeng $X$, Zhang Y, Kwong JSW, et al. The methodological quality assessment tools for preclinical and clinical studies, systematic review and meta-analysis, and clinica practice guideline: a systematic review. J Evid Based Med 2015;8:2-10.

26 Rostom A, Dubé C, Cranney A, et al. Appendix D. Quality Assessment Forms [Internet]. Agency for Healthcare Research and Quality (US), 2004. Available: https://www.ncbi. nlm.nih.gov/books/NBK35156/ [Accessed 3 Dec 2018]

27 Castilho ECD, Reis AMM, Borges TL, et al. Potential drug-drug interactions and polypharmacy in institutionalized elderly patients in a public hospital in Brazil. J Psychiatr Ment Health Nurs 2018;25:3-13.

28 Cuentro V da S. Avaliação da utilização e segurança de medicamentos em pacientes idosos internados em um hospital universitário, 2013. Available: http://repositorio. ufpa.br/jspui/handle/2011/6213

29 Santos RMdos, Sette IMF, de Farias Belém L. Drug use by elderly inpatients of a philanthropic Hospital. Braz J Pharm Sci 2011;47:391-8.

30 Pedroso FM. Prevalência de interações medicamentosas fármaco-fármaco em um hospital de Cuiabá, 2015. Available: https://sucupira.capes.gov.br/sucupira/public/ consultas/coleta/trabalhoConclusao/viewTrabalhoConclusao.jsf?popup=true\&id trabalho $=3600052$ [Accessed 5 Jun 2018].

31 de Oliveira, Lillian Rodrigues. Potenciais interações medicamentosas em prescrições de pacientes idosos internados no Hospital municipal de ouro preto do oeste - Ro, 2013. Available: https://sucupira.capes.gov.br/sucupira/public/consultas/coleta/ trabalhoConclusao/viewTrabalhoConclusao.jsf?popup=true\&id_trabalho=436398 [Accessed 5 Jun 2018].

32 Locatelli J. Interações medicamentosas em idosos hospitalizados. Einstein Säo Paulo 2007;5:343-6.

33 Melo D, Ribeiro E, Storpirtis S. Potential drug-drug interactions in a Brazilian teaching hospital: age-related differences? Rev Ciênc Farm Básica Apl 2015;36:435-44

34 Parreira RBC. Detecção de eventos adversos a medicamentos em idosos hospitalizados, 2016. Available: http://pesquisa.bvsalud.org/portal/resource/pt/tes6915? lang=fr [Accessed 5 Jun 2018].

35 Costa, Soraya Coelho. Avaliação da prescrição de medicamentos para idosos internados em Serviço de Clínica Médica do Sistema Único de Saúde em um hospital público universitário brasileiro, 2009. Available: http://www.bibliotecadigital.ufmg.br/ dspace/handle/1843/ECJS-7YYHUA [Accessed 5 Jun 2018].

36 Kashyap M, D'Cruz S, Sachdev A, et al. Drug-drug interactions and their predictors: results from Indian elderly inpatients. Pharm Pract 2013;11:191-5.

37 Danisha P, Dilip C, Mohan PL, et al. Identification and evaluation of potentially inappropriate medications (PIMs) in hospitalized geriatric patients using beers criteria. J Basic Clin Physiol Pharmacol J2015;26:403-10.

38 George AS, Ramesh M, Sebastian J. Adverse drug interactions in elderly hospitalized patients: a prospective analysis. Int J Pharm Sci Res 2018;9:1913-20.

39 Mohammed S, Poudel S, Laloo F, et al. Assessment of drug-related problems in a tertiary care teaching hospital, India. Asian J Pharm Clin Res 2017;10:310-3.

40 Devarapalli P, Soni S, T.K.N.V RK, et al. Assessment of inappropriate medication use in elderly inpatients of a tertiary care hospital in south-eastern India using the modified updated beers criteria 2003. Drugs Ther Perspect 2017:33:543-9.
41 Narvekar RS, Bhandare NN, Gouveia JJ, et al. Utilization pattern of potentially inappropriate medications in geriatric patients in a tertiary care hospital: a retrospective observational study. J Clin Diagn Res 2017;11:FC04-8.

42 Juárez-Cedillo T, Martinez-Hernández C, Hernández-Constantino A, et al. Clinical weighting of drug-drug interactions in hospitalized elderly. Basic Clin Pharmacol Toxicol 2016:118:298-305.

43 Rosas-Carrasco 0, García-Peña C, Sánchez-García S, et al. The relationship between potential drug-drug interactions and mortality rate of elderly hospitalized patients. Rev Investig Clin 2011;63:564-73.

44 Radošević N, Gantumur M, Vlahović-Palčevski V. Potentially inappropriate prescribing to hospitalised patients. Pharmacoepidemiol Drug Saf 2008;17:733-7.

45 Matanović SM, Vlahović-Palčevski V. Potentially inappropriate prescribing to the elderly: comparison of new protocol to beers criteria with relation to hospitalizations for ADRs. Eur J Clin Pharmacol 2014;70:483-90.

46 Chahin RC. Drug interactions in hospitalized patients in the internal medicine hospital university "Dr. Angel Larralde". June 2014 - December 2015. Arch Venez Farmacol Ter 2015;34:21-6

47 Janković SM, Pejčić AV, Milosavljević MN, et al. Risk factors for potential drug-drug interactions in intensive care unit patients. J Crit Care 2018:43:1-6.

48 Sapkota S, Pudasaini N, Singh C, et al. Drug prescribing pattern and prescription error in elderly: a retrospective study of inpatient record. Asian J Pharm Clin Res 2011;4:83-6.

49 Rafiei H, Esmaeili Abdar M, Moghadasi J. The prevalence of potential drug interactions among critically ill elderly patients in the intensive care unit (ICU). Iran J Ageing 2012;6:14-19.

50 Teka F, Teklay G, Ayalew E, et al. Potential drug-drug interactions among elderly patients admitted to medical ward of Ayder Referral Hospital, Northern Ethiopia: a cross sectional study. BMC Res Notes 2016;9:431.

51 Senín LD. Estudio sobre la utilización inapropiada de medicamentos en pacientes de edad avanzada hospitalizados en el servicio de medicina interna de un hospital privado, 2016. Available: http://rabida.uhu.es/dspace/bitstream/handle/10272/ 12099/Estudio_sobre_la_utilizacion_inapropiada.pdf?sequence=2 [Accessed 5 Jun 2018].

52 Gutiérrez-Valencia M, Izquierdo M, Malafarina V, et al. Impact of hospitalization in an acute geriatric unit on polypharmacy and potentially inappropriate prescriptions: a retrospective study. Geriatr Gerontol Int 2017:17:2354-60.

53 Blix HS, Viktil KK, Reikvam A, et al. The majority of hospitalised patients have drugrelated problems: results from a prospective study in general hospitals. Eur J Clin Pharmacol 2004;60:651-8.

54 Correia LM, Barros A, Brazão ML. Polifarmácia, Fármacos Inapropriados E Interacções Medicamentosas nas Prescrições de Doentes Nonagenários. Med Interna 2017;24:24-9

55 Egger T, Dormann $\mathrm{H}$, Ahne $\mathrm{G}$, et al. Identification of adverse drug reactions in geriatric inpatients using a computerised drug database. Drugs Aging 2003;20:769-76.

56 Ghibelli S, Marengoni A, Djade CD, et al. Prevention of inappropriate prescribing in hospitalized older patients using a computerized prescription support system (INTERcheck®). Drugs Aging 2013;30:821-8.

57 Schuler J, Dückelmann C, Beindl W, et al. Polypharmacy and inappropriate prescribing in elderly internal-medicine patients in Austria. Wien Klin Wochenschr 2008;120:733-41.

58 Barak-Tsafrir 0, Tellem R, Cialic R. Medications reconciliation to detect drug-drug interactions (DDI) - impact on medication regimen during geriatric hospitalization. $J$ Am Geriatr Soc 2016:64:S73-4.

59 Yong TY, Lau SY, Li JY, et al. Medication prescription among elderly patients admitted through an acute assessment unit. Geriatr Gerontol Int 2012:12:93-101.

60 Bundhun PK, Teeluck AR, Bhurtu A, et al. Is the concomitant use of clopidogrel and proton pump inhibitors still associated with increased adverse cardiovascular outcomes following coronary angioplasty?: a systematic review and meta-analysis of recently published studies (2012 - 2016). BMC Cardiovasc Disord 2017:17:3.

61 Johnston SC, Easton JD, Farrant M, et al. Clopidogrel and aspirin in acute ischemic stroke and high-risk TIA. N Engl J Med 2018;379:215-25.

62 Roblek T, Vaupotic T, Mrhar A, et al. Drug-drug interaction software in clinical practice: a systematic review. Eur J Clin Pharmacol 2015;71:131-42.

63 By the American Geriatrics Society 2015 Beers Criteria Update Expert Panel. American Geriatrics Society 2015 updated Beers criteria for potentially inappropriate medication use in older adults. J Am Geriatr Soc 2015;63:2227-46.

64 Tilson H, Hines LE, McEvoy G, et al. Recommendations for selecting drug-drug interactions for clinical decision support. Am J Health Syst Pharm 2016;73:576-85.

65 Roblek T, Trobec K, Mrhar A, et al. Potential drug-drug interactions in hospitalized patients with chronic heart failure and chronic obstructive pulmonary disease. Aoms 2014;5:920-32.

66 Peng CC, Glassman PA, Marks IR, et al. Retrospective drug utilization review: incidence of clinically relevant potential drug-drug interactions in a large ambulatory population. J Manag Care Pharm 2003;9:513-22.

67 Young T, Hopewell S, Cochrane Methodology Review Group. Methods for obtaining unpublished data. Cochrane Database Syst Rev 2011:22. 